\title{
Multi-criteria performance assessment based on closed-loop system identification
}

This paper was downloaded from TechRxiv (https://www.techrxiv.org).

\section{LICENSE}

CC BY 4.0

SUBMISSION DATE / POSTED DATE

$15-10-2021 / 26-10-2021$

CITATION

Sanchez, Gustavo (2021): Multi-criteria performance assessment based on closed-loop system identification. TechRxiv. Preprint. https://doi.org/10.36227/techrxiv.16817551.v1

DOI

10.36227/techrxiv.16817551.v1 


\title{
Multi-criteria performance assessment based on closed-loop system identification
}

\author{
Gustavo Sanchez \\ Department of Electrical and Electronics Engineering, Institute of Engineering and \\ Technology, JK Lakshmipat University, Jaipur, India. Address all correspondence to: \\ gustavo.sanchez@jklu.edu.in
}

\begin{abstract}
A method to assess the performance of control loops, based on closed-loop system identification is proposed. This method allows to take into account the trade-off between process variable and manipulated variable energy, thus overcoming one of the most important criticisms to Harris'index. To illustrate the proposed approach, a numerical example is given, for which the proposed index is $8 \%$ greater than Harris'index.
\end{abstract}

Keywords: Control Loop, Performance Assessment

\section{Introduction}

2 In the process industry sector, companies need to extract actionable information from sensor-based data collected on a daily basis through their integrated

4 IT/OT systems [1]. It is estimated that each operator typically needs to assess the performance of 90-180 control loops [2], and therefore the interest in

6 developing computer-aided tools to facilitate their work. In this framework, timely and accurate assessment may enable the implementation of appropriate 8 corrective actions as soon as required.

A common approach for control loop performance assessment (CPLA) is based on selecting an ideal benchmark to which the actual loop can be compared [3. As an example, if the Minimum Variance (MV) control loop is selected, it 12 is possible to determine the well-known Harris' index (HI), using only routine 
data, collected during normal operation, without the need of ad hoc invasive exloop performance $\mathcal{P}$ to be a discrete variable

$$
\mathcal{P} \in\{\text { Good, Fair, Poor }\}
$$

36 and extended to assess more complex processes [5].

Although numerous of similar techniques are available [6], due to real-world phenomena like non-linearity, unknown disturbances and dynamics, unstable or multivariable loops, etc., this can still be a challenging problem. In this paper we propose a multi-criteria approach based on the following rationales:

- It may be necessary to consider different aspects related to loop performance, possibly in contradiction, which is not possible using the classic HI. is too aggressive. Therefore, in practice it may largely underestimate loop performance.

- Companies are currently implementing, or willing to implement, computing infrastructure able to run more complex analysis algorithms, compared to past situation.

The structure of this paper is as follows. In section 2, problem statement is presented. In section 3, the proposed approach is described and in section 4 a simple case study is presented.

\section{Problem statement}

Let $\mathcal{S}$ be a commissioned stable single-input single-output closed-loop LTI system, as in Fig. 1, where $\varepsilon(k)$ is a disturbance assumed to be unit white noise.

Based on the data collected during the lapse $k=0,1,2, \ldots, N-1$, we define the

Thus, the plant under control is described by the following model

$$
\begin{aligned}
y(k) & =\frac{G_{p}\left(z^{-1}\right)}{1+G_{c}\left(z^{-1}\right) G_{p}\left(z^{-1}\right)} \varepsilon(k) \\
G_{p}\left(z^{-1}\right) & =\frac{B\left(z^{-1}\right)}{A\left(z^{-1}\right)}
\end{aligned}
$$




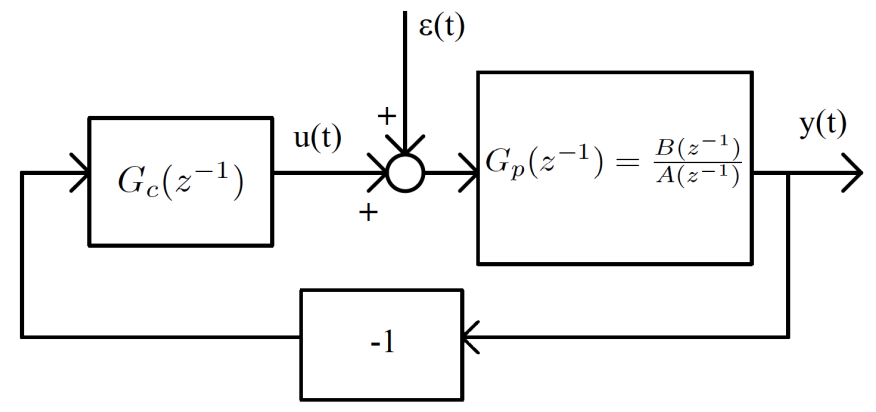

Figure 1: Commissioned control system

We assume that $A\left(z^{-1}\right), B\left(z^{-1}\right)$ are unknown polynomials, and $n, m$ their corresponding degrees. The controller model $G_{c}\left(z^{-1}\right)$ is assumed known. We are interested in the following problem: given $\mathcal{S}$ determine its performance $\mathcal{P}$, by using only the available measurements

$$
y\left(-N_{H}\right) \ldots, y(-2), y(-1), y(0), y(1), y(2), \ldots, y(N-1)
$$

where $N_{H}+N$ is the total number of available measurements, with $N_{H} \gg N$.

42

As discussed in the previous section, the classical approach to solve this problem is to determine the Harris' index (HI), denoted hereafter $\eta_{M V}$. If we

44 denote $\sigma_{M V}^{2}$ the minimum variance achievable by any linear controller, $\eta_{M V}$ will be given by the ratio:

$$
\eta_{M V}=\frac{\sigma_{M V}^{2}}{s_{y}^{2}}
$$

${ }_{46} \quad$ where $s_{y}^{2}$ is the measured output variance

$$
\begin{gathered}
s_{y}^{2}=\frac{1}{N-1} \sum_{k=0}^{N-1}\left[y(k)-m_{y}\right]^{2} \\
m_{y}=\frac{1}{N} \sum_{k=0}^{N-1} y(k)
\end{gathered}
$$

48 The estimation of minimum variance $\sigma_{M V}^{2}$ requires the identification of an ARMA model (see for example [3])

$$
\widehat{A}_{c l}\left(z^{-1}\right) y(k)=\widehat{B}_{c l}\left(z^{-1}\right) \varepsilon(k)
$$


from historical data $y\left(-N_{H}\right) \ldots, y(-2), y(-1), y(0)$, which allows also to estimate the closed-loop transfer function, given by

$$
\widehat{G}_{c l}\left(z^{-1}\right)=\frac{\widehat{B}_{c l}\left(z^{-1}\right)}{\widehat{A}_{c l}\left(z^{-1}\right)}
$$

52 Knowing $\sigma_{M V}^{2}$ and $\eta_{M V}$ (see equation 5) the loop performance $\mathcal{P}$ is given by

$$
\mathcal{P}=\left\{\begin{array}{c}
\text { Good, if } \eta_{M V} \geq \eta_{G} \\
\text { Fair, if } \eta_{P}<\eta_{M V}<\eta_{G} \\
\text { Poor, if } \eta_{M V} \leq \eta_{P}
\end{array}\right.
$$

54 where $\eta_{G}, \eta_{P}$ define performance ranges, usually determined by human experts according to their past experience and a priori information.

56

The previous approach is well-known and statistical confidence intervals can be calculated for $\eta_{M V}$ [7]. However, as discussed in the previous section, in ${ }_{58}$ practice we may be largely underestimating loop performance.

\section{Proposed approach}

60

We focus on the trade-off between process variable $y(k)$ and manipulated variable $u(k)$ energy. For this, let's denote

$$
m_{u}=\frac{1}{N} \sum_{k=0}^{N-1} u(k)
$$

62

$$
\begin{gathered}
s_{u}^{2}=\frac{1}{N-1} \sum_{k=0}^{N-1}\left[u(k)-m_{u}\right]^{2} \\
s_{y, u}=\left(s_{y}^{2}, s_{u}^{2}\right)
\end{gathered}
$$

As in the previous section, we start by estimating $\widehat{A}_{c l}\left(z^{-1}\right), \widehat{B}_{c l}\left(z^{-1}\right)$ (see equation 9 from which we obtain the open-loop transfer function

$$
\widehat{G}_{p}\left(z^{-1}\right)=\frac{\widehat{G}_{c l}\left(z^{-1}\right)}{1-G_{c}\left(z^{-1}\right) \widehat{G}_{c l}\left(z^{-1}\right)}=\frac{\widehat{B}\left(z^{-1}\right)}{\widehat{A}\left(z^{-1}\right)}
$$


${ }_{64}$ Note that in practice, the order of polynomials $\widehat{A}\left(z^{-1}\right), \widehat{B}\left(z^{-1}\right)$, namely $\hat{n}, \hat{m}$, needs to be assumed. Then, let $\hat{y}(k+\tau)$ be the one-step-ahead output prediction:

$$
\hat{y}(k+1)=\frac{F\left(z^{-1}\right)}{\widehat{B}\left(z^{-1}\right)} y(k)+E\left(z^{-1}\right) u(k)
$$

${ }_{66}$ where $E\left(z^{-1}\right)$ and $F\left(z^{-1}\right)$ are obtained solving the Diophantine equation

$$
\widehat{B}\left(z^{-1}\right)=E\left(z^{-1}\right) \widehat{A}\left(z^{-1}\right)+z^{-1} F\left(z^{-1}\right)
$$

For each value $\alpha=\frac{1}{N_{P}}, \frac{2}{N_{P}}, \cdots, 1$, with $N_{P} \gg 1$, we determine the solution ${ }_{68} u^{*}(k)$ to the following optimization problem

$$
\begin{aligned}
& \min \\
& u(k)
\end{aligned}\left[\alpha \hat{y}(k+1)^{2}+(1-\alpha) u(k)^{2}\right]
$$

Here, it is possible to show that the solution is $u^{*}(k)$ such that

$$
u^{*}(k)=-K_{\alpha}^{*}\left(z^{-1}\right) y(k)
$$

70 with

$$
K_{\alpha}^{*}\left(z^{-1}\right)=\frac{\alpha F\left(z^{-1}\right) E\left(z^{-1}\right)}{\alpha E\left(z^{-1}\right)^{2} B\left(z^{-1}\right)+(1-\alpha) B\left(z^{-1}\right)}
$$

Note that this result (18) was found independently by the author of this 72 paper, however an equivalent result was previously published in 8 . Now, denote $S^{*}$ the set of points $\left(s_{u, \alpha}^{* 2}, s_{y, \alpha}^{* 2}\right)$

$$
s_{u, \alpha}^{* 2}=\left\|\frac{G_{p}\left(z^{-1}\right) K_{\alpha}^{*}\left(z^{-1}\right)}{1+K_{\alpha}^{*}\left(z^{-1}\right) G_{p}\left(z^{-1}\right)}\right\|_{2}^{2}
$$

74

$$
s_{y, \alpha}^{* 2}=\left\|\frac{G_{p}\left(z^{-1}\right)}{1+K_{\alpha}^{*}\left(z^{-1}\right) G_{p}\left(z^{-1}\right)}\right\|_{2}^{2}
$$

for $\alpha=\frac{1}{N_{P}}, \frac{2}{N_{P}}, \cdots, 1$. Note that $S^{*}$ is a numerical approximation of the Pareto 76 front [9] corresponding to the problem

$$
\min _{u(k)}\left(\begin{array}{c}
u(k)^{2} \\
\hat{y}(k+1)^{2}
\end{array}\right)
$$


Now, denote $\left(p_{u, \alpha}^{* 2}, p_{y, \alpha}^{* 2}\right)$ the point in $S^{*}$ which is nearest to $s_{y, u}=\left(s_{y}^{2}, s_{u}^{2}\right)$

but dominates $s_{y, u}$, which means

$$
\begin{aligned}
& p_{u, \alpha}^{* 2} \leq s_{u}^{2} \\
& p_{y, \alpha}^{* 2} \leq s_{y}^{2}
\end{aligned}
$$

80

The performance will be assessed based on the following indicator:

$$
\eta_{M C}=\frac{p_{y, \alpha}^{* 2}}{s_{y}^{2}}
$$

Note inmediately that

$$
p_{y, \alpha}^{* 2} \geq \sigma_{M V}^{2}
$$

and therefore

$$
\eta_{M C} \geq \eta_{M V}
$$

\section{Numerical results}

Consider the first-order system described by

$$
y(k)=\frac{1}{1+a_{1} z^{-1}}[u(k)+\varepsilon(k)]
$$

${ }_{84}$ with $a_{1}=-0.9$ (also considerd in [3]) and controller

$$
G_{c}\left(z^{-1}\right)=\frac{0.2 z^{-1}}{1-0.8999 z^{-1}}
$$

As explained in the previous section, first we generate the data

$$
y\left(-N_{H}\right) \ldots, y(-2), y(-1)
$$

${ }_{86}$ and identify the following third-order ARMA model, assuming $\hat{n}=3, \hat{m}=3$,

$$
\widehat{G}_{c l}\left(z^{-1}\right)=\frac{\widehat{B}_{c l}\left(z^{-1}\right)}{\widehat{A}_{c l}\left(z^{-1}\right)}=\frac{1-0.7863 z^{-1}-0.0629 z^{-2}-0.0298 z^{-3}}{1-1.743 z^{-1}+0.620 z^{-2}+0.09022 z^{-3}}
$$

and generate $S^{*}$ showed in Fig. 22 with $N_{P}=30$, and

$$
K_{\alpha}^{*}\left(z^{-1}\right)=\alpha \frac{0.904-1.504 z^{-1}+0.505 z^{-2}+0.1020 z^{-3}}{1-0.7863 z^{-1}-0.0629 z^{-2}-0.0298 z^{-3}}
$$


88 Note that the distribution of points (black dots) is not uniform. The red line corresponds to the interpolation curve corresponding to the Pareto approximation 9o obtained by using the real polynomials $A\left(z^{-1}\right)$ and $B\left(z^{-1}\right)$.

Next, we generate the data

$$
y(0), y(1), y(2), \ldots, y(N-1)
$$

which corresponds to the window we want to assess, obtaining the following 92 results

$$
\begin{aligned}
\left(s_{y}^{2}, s_{u}^{2}\right) & =(2.74,0.5) \\
\left(p_{u, \alpha}^{* 2}, p_{y, \alpha}^{* 2}\right) & =(1.22,0.28) \\
\eta_{M V}(\%) & =36.5 \\
\eta_{M C}(\%) & =44.4
\end{aligned}
$$

Note, that, as expected, the proposed multi-criteria index is almost $8 \%$ greater than Harris' index. The code to obtain these results is available in the following link: https://github.com/multiopti/research/blob/main/MC_CLPA.ipynb

\section{Conclusion}

A multi-criteria approach for control loop performance assessment was proposed, which allows to take into account the trade-off between process variable and manipulated variable energy, thus overcoming one of the most important criticisms to Harris'index. In future, we plan to extend this method to more complex models, including unstable multivariable processes.

\section{References}

[1] R. Bacci di Capaci, C. Scali, A Cloud-Based Monitoring System for Performance Assessment of Industrial Plants, Industrial \& Engineering Chemistry Research 59 (6) (2020) 2341-2352. doi:10.1021/acs.iecr.9b06638

URL https://pubs.acs.org/doi/10.1021/acs.iecr.9b06638 


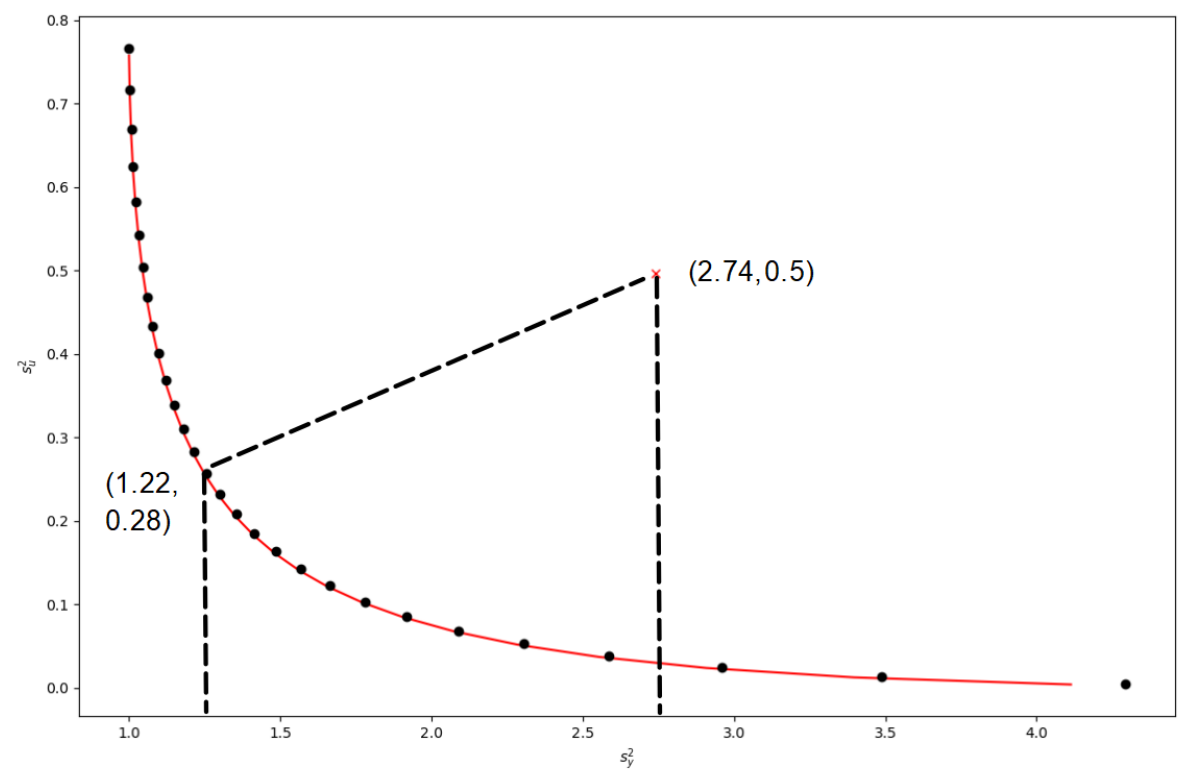

Figure 2: Pareto approximation

[2] R. Rys, Actionable Data Analytics for Control Engineers (Jul. 2016).

URL https://www . automationworld.com/factory/iiot/blog/ 13315771/actionable-data-analytics-for-control-engineers

[3] M. Jelali, Control Performance Management in Industrial Automation: Assessment, Diagnosis and Improvement of Control Loop Performance, Springer, 2013.

[4] Harris, Assessment of Control Loop Performance, The Canadian Journal of Chemical Engineering 69 (1989) 856-861.

[5] M. L. Tyler, M. Morari, Performance monitoring of control systems using likelihood methods, in: Proceedings of 1995 American Control Conference ACC'95, Vol. 2, 1995, pp. 1245-1249 vol.2. doi:10.1109/ACC.1995.520949

[6] S. J. Qin, Survey on data-driven industrial process monitoring and diagnosis, Annual Reviews in Control 36 (2) (2012) 220 - 234. 
doi:https://doi.org/10.1016/j.arcontrol.2012.09.004

URL

http://www.sciencedirect.com/science/article/pii/

[7] T. J. Harris, Statistical properties of quadratic-type performance indices, Journal of Process Control 14 (8) (2004) 899-914. doi:10.1016/j.jprocont.2004.02.006.

URL

https://linkinghub.elsevier.com/retrieve/pii/ S095915240400023X

${ }_{128} \quad[8]$ M. J. Grimble, Controller performance benchmarking and tuning using generalised minimum variance control, Automatica 38 (12) (2002) 2111-2119.

130 doi:10.1016/S0005-1098(02)00141-3.

a URL https://www.sciencedirect.com/science/article/pii/

${ }_{132} \quad$ S0005109802001413

[9] G. Sánchez, M. Villasana, M. Strefezza, Multi-objective Pole Placement with Evolutionary Algorithms, in: S. Obayashi, K. Deb, C. Poloni, T. Hiroyasu, T. Murata (Eds.), Evolutionary Multi-Criterion Optimization, Springer Berlin Heidelberg, Berlin, Heidelberg, 2007, pp. 417-427. 